\title{
Influence of Ligation Methods on Plaque Accumulation and White Spots Formation around Orthodontic Brackets
}

\author{
Jun H Park ${ }^{1}$, Peter T Gakunga ${ }^{1}$, Lakshmi Vishwanat ${ }^{2}$ and Bennett T Amaechi ${ }^{2 *}$ \\ ${ }^{1}$ Department of Developmental Dentistry, The University of Texas Health Science Center, USA \\ ${ }^{2}$ Department of Comprehensive Dentistry, The University of Texas Health Science Center, USA
}

Received: February 02, 2014; Accepted: February 18, 2014; Published: February 21, 2014

*Corresponding author: Bennett T Amaechi, Department of Comprehensive Dentistry, University of Texas Health Science Center, San Antonio, 7703 Floyd Curl Drive, San Antonio, Texas 78229-3900, USA, Tel: 1-210-567-3185; Fax: 1-210-567-4587; E-mail: amaechi@uthscsa.edu

\begin{abstract}
Objectives: The formation of white spot lesion around orthodontic brackets during treatment is a significant clinical and esthetic problem. This has been associated with plaque formation around the brackets. Plaque retention around orthodontic appliances has been attributed to several factors including archwire ligation method. The purpose of this study was to investigate the influence of ligation method on plaque accumulation and demineralization around brackets. We hypothesized that self-ligating brackets would accumulate less plaque and cause less demineralization than conventional brackets with elastomeric ligatures.
\end{abstract}

Methods: 45 enamel blocks were randomly assigned to 3 groups: Edgewise $^{\circledR}$ brackets (ringless); self-ligating Speed ${ }^{\circledR}$ brackets (selfligating); Edgewise ${ }^{\circledR}$ brackets with elastomeric rings (e-ringed). The blocks were subjected to a plaque-forming cariogenic environment using an artificial mouth system in a mixed-organisms continuous flow biofilm model simulating oral conditions. Using quantitative light-induced fluorescence, plaque was measured for 5 consecutive days and demineralization was quantified after 28 days.

Results: A significant increase $(\mathrm{p}<0.001)$ in plaque accumulation were observed between days 1 and 5 in all groups, but the differences among the groups were not statistically significant. Demineralization was significantly higher in e-ringed compared to ringless $(\mathrm{p}=0.0016)$ and self-ligating $(\mathrm{p}=0.0009)$ brackets. There was no significant difference between ringless and self-ligating brackets, demonstrating that bracket design was not responsible for the difference in demineralization but the presence of elastomeric ring.

Conclusions: Ligation using elastomeric rings is associated with increased demineralization around orthodontic brackets.

Keywords: Ligation; Plaque; White spots; Orthodontic brackets; Demineralization

\section{Introduction}

Early caries lesions in enamel appear clinically as opaque white spot lesions [1-5]. These lesions indicate demineralization of enamel caused by acids produced by cariogenic microorganism in plaque [6-9]. The formation of white spot lesion around orthodontic brackets during treatment is a significant clinical and esthetic problem [3,4,10-13]. Although some researchers have investigated some possibilites relating to this, the causative role played by the method of bracket ligation to archwire has not been adequately evaluated.

Elastomeric rings are commonly used in orthodontics to hold the archwire in the bracket slot. They are easy to apply, comfortable to the patient, and available in a variety of colors, making them popular among young patients. However, elastomeric rings promote plaque formation by accumulating more micro-organisms compared to stainless steel ligatures [14]. The plaque and saliva of patients undergoing orthodontic treatment with standard edgewise appliances ligated with elastomeric rings contain a higher prevalence of $S$. mutans compared to those with steel ligatures [15-17].

Self-ligating brackets do not require elastomeric rings. The ligating mechanism is built into the bracket system. These brackets are associated with improved treatment efficiency, significantly fewer appointments, and better quality of treatment outcomes compared to standard edgewise appliances [18-21]. Although it has been suggested that self-ligating brackets also promote better oral hygiene [22], no scientific studies have compared self-ligating and conventional orthodontic brackets with respect to plaque accumulation or demineralization during orthodontic treatment.

In this study we quantitatively monitored plaque formation and assessed demineralization around different orthodontic brackets. Several methods can detect early demineralization on enamel surface. These include visual inspection [23,24], electronic caries monitoring [25,26], fiber-optic transillumination (FOTI) [27], laser fluorescence (DIAGNOdent) [28], and transverse microradiography (TMR) $[29,30]$. In this study, we used quantitative light fluorescence (QLF), a nondestructive, sensitive, reliable, and reproducible method [31-34]. QLF has been validated against TMR, which is considered the in vitro gold standard, on both permanent and deciduous teeth [35]. Moreover, QLF in vitro has high inter-examiner reliability [36]. Amaechi and Higham [31] established the optimum conditions for the use of QLF. Recent development in this system incorporates a red fluorescence detector; excitation of red extrinsic fluorophores from bacterial metabolites 
(porphyrins) by blue light causes plaque, calculus, and bacteria-infected caries lesions to appear red on a bright green background, enabling the use of QLF to quantify and monitor plaque [37].

The purpose of this study was to compare the rate of plaque accumulation and the degree of demineralization in vitro in enamel blocks on which the following types of brackets were bonded, either selfligating brackets or brackets ligated with elastomeric rings, or brackets with no ligation at all. We hypothesized that self-ligating brackets would accumulate less plaque and cause less demineralization than conventional brackets with elastomeric ligatures. We also evaluated conventional brackets without elastomeric ligatures to distinguish the specific effects of the elastomeric ligatures from differences in bracket design and size.

\section{Materials and Methods}

\section{Teeth preparation and randomization}

Freshly extracted and appropriately disposed sound human molars and premolars were collected. Teeth were extracted either for orthodontic or for third-molar impaction reasons in various UTHSCSA Dental School clinics. The teeth were cleaned of debris/stains, and examined. Fifteen teeth without caries, cracks or enamel malformations were selected and cleaned with pumice to remove the remnants of pellicle. Using Well's diamond cutting saw (Well's Company, Germany), three enamel blocks, approximately $5.0 \mathrm{~mm}^{2}$ were produced from each tooth, giving a total of 45 enamel blocks. The enamel blocks were randomly assigned to three experimental groups (15 blocks/group): (A) Edgewise ${ }^{\circledR}$ brackets without elastomeric rings (control), (B) Self-ligating Speed ${ }^{\circledR}$ brackets, and (C) Edgewise ${ }^{\circledR}$ brackets with elastomeric rings. The brackets were bonded to their respective blocks (Figure 1) using Transbond ${ }^{\circledR}$ XT light-cure adhesive paste (3M Unitek, St Paul, MN). The remaining enamel surface of each block was then painted with two coats of non-fluorescent colorless acid resistant nail varnish except for a ring of exposed enamel (extending approximately $1 \mathrm{~mm}$ from the edge of the bracket) around the bracket, created using an adhesive tape. The ring of exposed enamel was created in all samples using the same adhesive tape to ensure uniform area of exposed enamel surface.

\section{Experimental procedure}

The experiment was conducted in a continuous flow biofilm model acting as an artificial mouth. This is a multiple-chamber continuous flow culture system described in our previous studies (Figure 2) [38]. The system is housed inside a $\mathrm{CO}_{2}$ incubator maintained at a constant physiological temperature of $37^{\circ} \mathrm{C}$. The bracket-bearing blocks were randomly assigned to three culture chambers in the artificial mouth system (15 blocks/chamber) irrespective of their group. Using heavy duty putty, these blocks were embedded in the vertical grooves on the surface of the cylindrical rod inside the culture chamber. These blocks were embedded such that their surfaces flushed with the surface of the cylinder to permit streamlined flow of fluids, and the exposed enamel was available for plaque growth and subsequent demineralization. Prior to the experiment, all components of the Artificial Mouth including the teeth were sterilized using ethylene oxide gas. White spot lesions development on the tooth surfaces were initiated by inoculation of the chambers by 1-hour circulation of mixed Streptococcus mutans (NCTC 10449) and Lactobacilli casei (NCIB 8820) culture in Todd Hewitt broth (broth to inoculums ratio 10:1) through the chambers. Inoculation was repeated

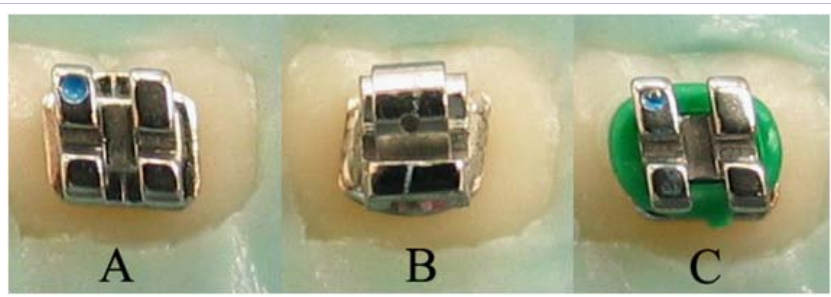

Figure 1: Experimental Groups.

Group A: Edgewise bracket without any ligation; Group B: Self-ligating Speed bracket; Group C: Edgewise bracket with elastomeric ring.

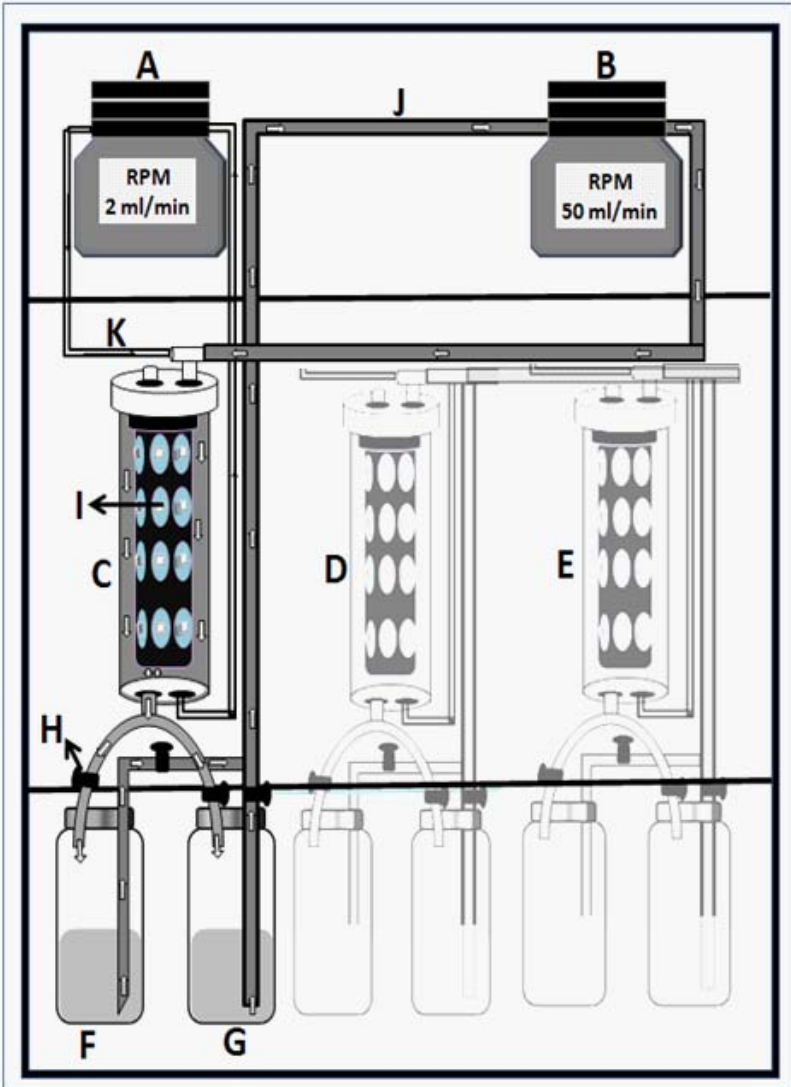

Figure 2: Schematic representation of the artificial mouth setup in $\mathrm{CO}_{2}$ incubator. A- Programmable filling \& emptying pump; B- Programmable broth and sucrose circulation pump; C, D \& E- Oral chamber housing experimental teeth; F- Todd Hewitt broth; G- Sucrose; H- Liquid controlling stopper; I- Artificial jaw with tooth blocks; J- Broth and sucrose pumping tube; K- Broth circulating tube.

once daily for two consecutive days. Todd Hewitt broth was continuously and simultaneously supplied to the four chambers to simulate saliva, while $5 \%$ sucrose was supplied every 6 hours for 6 minutes to simulate meals. All fluids, including inoculation, were delivered at a flow rate of 0.3 $\mathrm{ml} / \mathrm{min}$ (average unstimulated salivary flow rate). Change in plaque $\mathrm{pH}$ following sucrose supply was monitored on two occasions on the third day to confirm exhibition of Stephan's curve (plaque pH curve) under sucrose challenge. A micro-esophageal glass $\mathrm{pH}$ electrode and microreference electrode connected through a pH meter were installed in each chamber at the plaque growth surface to monitor the intra-plaque $\mathrm{pH}$. 

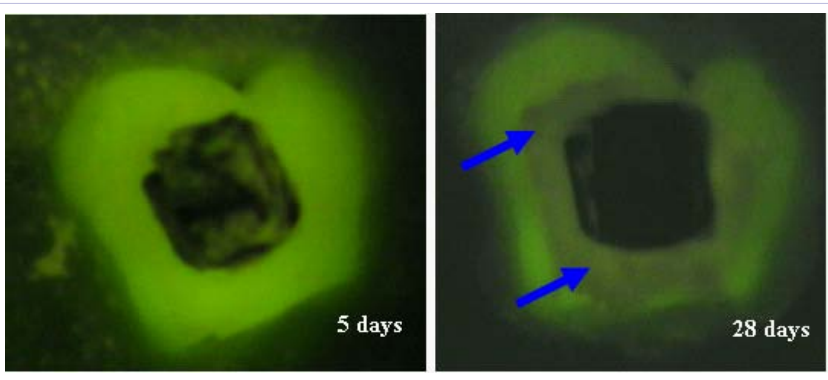

Figure 3: QLF images before exposure to plaque (0 days) and after 28 days of plaque accumulation. Note the demineralization (fluorescence loss) around the bracket after 28 days (arrows).

\section{Plaque and demineralization quantification}

Plaque accumulation on the enamel surfaces was detected and measured daily for the five days using quantitative light-induced fluorescence $\left(Q_{L F}{ }^{\mathrm{TM}}\right)$. Plaque appears as red fluorescence on enamel surface when viewed with QLF and is quantified as red fluorescence intensity $(\Delta \mathrm{R})$. Following 5 days measuring of plaque the experiment was continued for 28 days for development of white spot lesions (demineralization). At the end of 28 days, each enamel block was brushed with a soft-bristled toothbrush to remove all the plaque. Plaque interferes with QLF imaging by masking the caries lesion under the plaque. Following brushing, the tooth surface was dried with an air syringe for 5 seconds, which revealed clearly the white spot lesions around the brackets (Figure 3). This was followed by QLF imaging of the enamel blocks by a single examiner. White spot lesions appear as dark spots (area of reduced fluorescence) when viewed with QLF (Figure 3).

The QLF system comprised of a special intra-oral micro-camera device connected to a computer fitted with a frame grabber (Comet, Matrox, Electronic systems Ltd, Quebec, Canada) and to which the QLF software (QLF version 2.0.37, Inspektor Research Systems BV, Amsterdam, The Netherlands) was installed. To visualize and capture the tooth image, white light from a special arc lamp (Philips bv, Eindhoven, The Netherlands) based on Xenon technology was filtered through a bluetransmitting bandpass filter (Philips bv, Eindhoven, The Netherlands) with peak intensity of $\lambda=370 \mathrm{~nm}$ and bandwidth of $80 \mathrm{~nm}$, to provide illumination of the tooth with a blue-violet light with an intensity of 13 $\mathrm{mW} / \mathrm{cm}^{2}$. A dental mirror provided uniform illumination of the tooth, and with the aid of a color CCD-sensor (Sony LS-1P, Tokyo, Japan), which had a yellow-transmitting $(\lambda \geq 520 \mathrm{~nm})$ filter (Philips bv, Eindhoven, The Netherlands) positioned in front of it (to filter out all reflected and backscattered light), the fluorescent image of the tooth was recorded and digitized by the framegrabber and was available for quantitative analysis with the QLF software as described by de Josselin de Jong [39], The QLF software automatically gives the value for the decrease in fluorescence (demineralization), $\Delta \mathrm{Q}\left(\% \cdot \mathrm{mm}^{2}\right)$, for white spot lesions or red fluorescence intensity, $\Delta \mathrm{R}(\%)$, for plaque quantity, with a simultaneous data storage.

\section{Statistical analysis}

Statistical analysis of the data was performed with SPSS (version 14.0 , Chicago Illinois) with the level of significance ( $\alpha$ ) pre-chosen at 0.05 . For plaque accumulation analysis, mean values and standard deviations of $\Delta \mathrm{R}$ were calculated for each of the five days. Intra- and inter-group comparison of plaque accumulation over time was carried out using Bonferroni corrections to reduce the possibility of significances due to chance. Inter-group comparison of the levels of demineralization after 28 days was examined using Analysis of variance (ANOVA) with post-hoc Tukey's comparison tests.

\section{Results}

Table 1 shows the mean and standard deviation of plaque accumulation $(\Delta R)$ from day 1 to day 5 . Significant differences $(p<0.001)$ in mean $\Delta \mathrm{R}$ were observed in day 1 through day 5 in all three groups; however, the differences in mean $\Delta \mathrm{R}$ among the groups was not statistically significant on any day, but consistently ranked as follows in numerical value: edgewise E-ringed brackets > self-ligating brackets > edgewise ringless brackets (control). All three groups reached maximum plaque accumulation at day 3 , at which point there was a slight decrease.

Figure 4 shows the amount of demineralization $(\Delta Q)$ in each group after 28 days of plaque formation. Demineralization was significantly higher $(\mathrm{p}=0.0004)$ in brackets with elastomeric rings compared to both the self-ligating brackets and the control group. The amount of demineralization around self-ligating brackets was similar to that of the ringless control group $(\mathrm{p}=0.9)$.

\section{Discussion}

Brackets with elastomeric rings demonstrated greater plaque development and demineralization compared to self-ligated brackets and brackets without ligation. This finding is in agreement with previous studies demonstrating that elastomeric rings accumulate significantly more plaque than steel-ligatures [14]. Forsberg [14] reported that the lateral incisor ligated with an elastomeric ring had a greater number of microorganisms in plaque than teeth ligated with steel wire. They recommended that elastomeric ligation rings should not be used in patients with compromised oral hygiene, as the increased microbial accumulation adjacent to the brackets, could predispose to the development of dental caries and gingivitis. A recent clinical study also showed that teeth ligated with elastomeric rings were more prone to bleeding, and the authors recommend against the use of elastomeric rings in patients with poor oral hygiene [40].

Twenty-eight days of plaque formation was associated with distinctly

Table 1: Plaque Accumulation, Measured as Red Fluorescence Intensity $(\Delta \mathrm{R})$, for the Three Ligation Groups. Note: A significant increase $(\mathrm{p}<0.001)$ in plaque accumulation were observed between days 1 and 5 in all groups.

\begin{tabular}{|l|l|l|l|l|l|}
\hline Groups & $\begin{array}{l}\text { Day } \mathbf{1} \\
\text { Mean } \boldsymbol{\Delta R}(\boldsymbol{S D})\end{array}$ & $\begin{array}{l}\text { Day 2 } \\
\text { Mean } \boldsymbol{\Delta R}(\boldsymbol{S D})\end{array}$ & $\begin{array}{l}\text { Day 3 } \\
\text { Mean } \boldsymbol{\Delta R}(\boldsymbol{S D})\end{array}$ & $\begin{array}{l}\text { Day 4 } \\
\text { Mean } \Delta \boldsymbol{R}(\boldsymbol{S D})\end{array}$ & $\begin{array}{l}\text { Day } \mathbf{5} \\
\text { Mean } \Delta \boldsymbol{R}(\boldsymbol{S D})\end{array}$ \\
\hline Brackets without ligation & $23.43(2.23)$ & $23.56(1.91)$ & $23.77(2.62)$ & $23.18(1.42)$ & $22.29(1.30)$ \\
\hline Self-ligating brackets & $23.45(1.38)$ & $23.67(1.49)$ & $23.86(1.36)$ & $23.75(1.30)$ & $23.36(0.83)$ \\
\hline Brackets with elastomeric ring & $23.61(1.89)$ & $23.90(2.25)$ & $25.13(1.46)$ & $24.57(1.77)$ & $23.41(1.50)$ \\
\hline
\end{tabular}




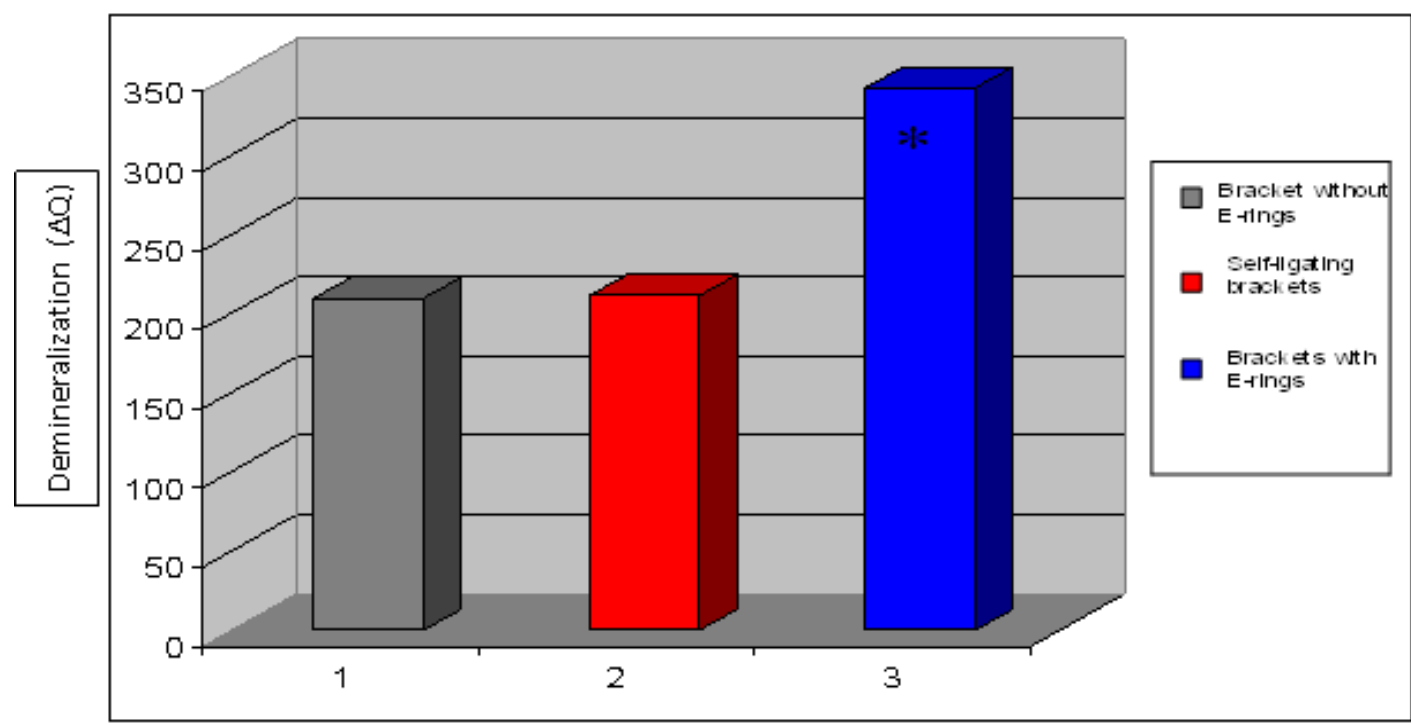

Experimental groups

Figure 4: Integrated fluorescence loss $(\Delta Q)$ for the 3 ligation methods. *significantly higher compared to $1(\mathrm{p}=0.0016)$ and 2 ( $\mathrm{p}=0.0009)$ brackets 1 and 2 .

more enamel demineralization around brackets ligated with elastomeric rings compared to self-ligated brackets and brackets without ligation. Although this is an in vitro study, this result is conceivable both in vitro and in vivo considering the fact that the external ligation from elastomeric ring would encourage more plaque accumulation, and even in vivo may limit oral hygiene efficiency around the bracket. Self-ligating bracket systems have a built-in mechanical lock, and thus do not require external ligation. Furthermore, the self-ligating and ligated brackets have a different base area, profile height, and other differences, which potentially might also affect plaque formation. However, the self-ligating brackets promoted a similar amount of demineralization as the Edgewise bracket without ligation, suggesting that the elastomeric ligatures and not differences in bracket design that was responsible for the increased demineralization. Therefore, our results support the hypothesis that the ligation method plays a significant role in the amount of demineralization.

Self-ligating brackets have been in the market since the 1930's, but due to cost and mechanical faults they have not been favored [22]. Today, a greater number of orthodontists are favoring these brackets due to their frictionless properties [41,42] and treatment efficiency [18-21]. The lack of evidence demonstrating that self-ligating brackets minimize white spot lesions led to the current study. This study proved that the amount of demineralization around these brackets is significantly smaller than around brackets with elastomeric rings. In addition to the external ligation method, the material composition of the elastomeric rings could have further contributed to the increased demineralization. Further studies comparing steel ligatures, elastomeric rings and fluoridated elastomeric rings are needed to determine whether the composition of elastomeric rings and/or the mechanical aspect of ligation are what determine increased demineralization.

The presence of plaque is an important factor in the development of white spot lesions. The use of an in vitro model to predict the interactions between dental plaque and orthodontic brackets is highly desirable, since this model controls for the variability, in clinical studies, when relying on patient compliance with oral hygiene. The artificial mouth system used in this study is particularly suited for studies of biofilms of oral bacteria in that it provides an environment similar to that found in the oral cavity. This model simulates oral cavity conditions such as temperature, salivary flow rate, bacterial colonization, and plaque formation, and therefore helps control as many confounding factors as possible, except tooth brushing. Although there are varieties of in vitro models acting as artificial mouth, this model has several advantages when compared to other systems $[43,44]$. The size of the acrylic cylinder simulating the oral surface and on which the samples are attached was design to the approximate the determined surface area of the oral cavity so that the amount of fluid on the surface at any time will approximate the amount of saliva in the adult mouth [45]. The continuous flow and recirculation of simulated oral fluids and bacterial consortium are delivered at a flow rate equal to the unstimulated salivary flow rate. Moreover, the plaque growth demonstrated the typical Stephan's curve with sucrose rinse, the characteristics of a cariogenic plaque. This system like previous systems [44] was able to produce an established cariogenic plaque within four days as confirmed by Stephan curve. Furthermore, a significant demineralization was produced in enamel within 28 days of plaque accumulation, which is in agreement with previous studies $[43,44]$.

An unexpected finding was that plaque grew progressively in all three groups, reaching a peak value on day 3; however, after day 4 the amount of plaque decreased. This decrease may have been due either to a limit to plaque thickness due to competition in microbial ecosystem or change in microbial composition with the aerobic microorganisms, Streptococcus mutans withering away with the increase in plaque thickness, while only the facultative anaerobes, Lactobacilli, survive the increase. This behavior may also have contributed to some extent to the lack of statistical differences observed between the groups. 
Considering the fact that this study excluded certain factors associated with a patient, such as toothbrushing and the protective functions of the saliva, this result should not be directly interpreted as what should be observed in a patient's situation. Although we are currently conducting a second in vitro study involving toothbrushing, steel ligature method and archwire, the true picture of the influence of ligation methods on white spot formation on patients undergoing orthodontic treatment can only be observed in a randomized clinical trial of the ligation methods.

\section{Conclusions}

Brackets with elastomeric rings encouraged non-significantly more plaque accumulation and significantly more demineralization compared to similar brackets without elastomeric rings or self-ligating brackets, indicating that the use of elastomeric rings as a ligating device encourages more plaque retention and consequent enamel demineralization.

\section{References}

1. Mizrahi E (1983) Surface distribution of enamel opacities following orthodontic treatment. Am J Orthod 84(4): 323-331.

2. Mitchell L (1992) Decalcification during orthodontic treatment with fixed appliances--an overview. Br J Orthod 19(3): 199-205.

3. Sectakof PA, Selnes JE (1994) Iatrogenic effects of orthodontic treatment. Ont Dent 71(9): 35-40.

4. Selnes JE, Sectakof PA (1994) Iatrogenic effects of orthodontic treatment. Part II: Demineralization. Univ Tor Dent J 7(1): 12-14.

5. Basdra EK, Huber H, Komposch G (1996) Fluoride released from orthodontic bonding agents alters the enamel surface and inhibits enamel demineralization in vitro. Am J Orthod Dentofacial Orthop 109(5): 466-472.

6. Chang HS, Walsh LJ, Freer TJ (1999) The effect of orthodontic treatment on salivary flow, $\mathrm{pH}$, buffer capacity, and levels of mutans streptococci and lactobacilli. Aust Orthod J 15(4): 229-234.

7. Wilson M (2001) Bacterial biofilms and human disease. Sci Prog 84(Pt3): 235-254.

8. Hicks J, Garcia-Godoy F, Flaitz C (2003) Biological factors in dental caries: role of saliva and dental plaque in the dynamic process of demineralization and remineralization (part 1). J Clin Pediatr Dent 28(1): 47-52.

9. Banas JA (2004) Virulence properties of Streptococcus mutans. Front Biosci 9: 1267-1277.

10. Tillery TJ, Hembree JH Jr, Weber FN (1976) Preventing enamel decalcification during orthodontic treatment. Am J Orthod 70(4): 435439 .

11. Gorelick L, Geiger AM, Gwinnett AJ (1982) Incidence of white spot formation after bonding and banding. Am J Orthod 81(2): 93-98.

12. Artun J, Brobakken BO (1986) Prevalence of carious white spots after orthodontic treatment with multibonded appliances. Eur J Orthod 8(4): 229-234.

13. Ogaard B, Rolla G, Arends J, ten Cate JM (1988) Orthodontic appliances and enamel demineralization. Part 2. Prevention and treatment of lesions. Am J Orthod Dentofacial Orthop 94(2): 123-128.

14. Forsberg CM, Brattstrom V, Malmberg E, Nord CE (1991) Ligature wires and elastomeric rings: two methods of ligation, and their association with microbial colonization of Streptococcus mutans and lactobacilli. Eur J Orthod 13(5): 416-420.
15. Mattingly JA, Sauer GJ, Yancey JM, Arnold RR (1983) Enhancement of Streptococcus mutans colonization by direct bonded orthodontic appliances. J Dent Res 62(12): 1209-1211.

16. Scheie AA, Arneberg P, Krogstad O (1984) Effect of orthodontic treatment on prevalence of Streptococcus mutans in plaque and saliva. Scand J Dent Res 92(3): 211-217.

17. Rosenbloom RG, Tinanoff N (1991) Salivary Streptococcus mutans levels in patients before, during, and after orthodontic treatment. Am J Orthod Dentofacial Orthop 100(1): 35-37.

18. Eberting JJ, Straja SR, Tuncay OC (2001) Treatment time, outcome, and patient satisfaction comparisons of Damon and conventional brackets. Clin Orthod Res 4(4): 228-234.

19. Harradine NW (2001) Self-ligating brackets and treatment efficiency. Clin Orthod Res 4(4): 220-227.

20. Marshall SD, Currier GF, Hatch NE, Huang GJ, Nah HD (2010) Ask us. Self-ligating bracket claims. Am J Orthod Dentofacial Orthop 138(2):128-131.

21. Chen SS, Greenlee GM, Kim JE, Smith CL, Huang GJ (2010) Systematic review of self-ligating brackets. Am J Orthod Dentofacial Orthop 137(6): 726.e1-726.e18.

22. Harradine NW (2003) Self-ligating brackets: where are we now? J Orthod 30(3): 262-273.

23. Ten Bosch JJ, Angmar-Mansson B (1991) A review of quantitative methods for studies of mineral content of intra-oral caries lesions. J Dent Res 70(1): 2-14.

24. Angmar-Mansson B, ten Bosch JJ (1993) Advances in methods for diagnosing coronal caries--a review. Adv Dent Res 7(2): 70-79.

25. Ashley PF, Blinkhorn AS, Davies RM (1998) Occlusal caries diagnosis: an in vitro histological validation of the Electronic Caries Monitor (ECM) and other methods. J Dent 26(2): 83-88.

26. Ashley PF, Ellwood RP, Worthington HV, Davies RM (2000) Predicting occlusal caries using the Electronic Caries Monitor. Caries Res 34(2): 201-203.

27. Hintze H, Wenzel A, Danielsen B, Nyvad B (1998) Reliability of visual examination, fibre-optic transillumination, and bite-wing radiography, and reproducibility of direct visual examination following tooth separation for the identification of cavitated carious lesions in contacting approximal surfaces. Caries Res 32(3): 204-209.

28. Ross G (1999) Caries diagnosis with the DIAGNOdent laser: a user's product evaluation. Ont Dent 76(2): 21-24.

29. Damen JJ, Exterkate RA, ten Cate JM (1997) Reproducibility of TMR for the determination of longitudinal mineral changes in dental hard tissues. Adv Dent Res 11(4): 415-419.

30. Benson PE, Higham SM, Pender N (2003) An in vitro assessment using transverse microradiography of the effect on mineral loss of etching enamel for in situ studies. Orthod Craniofac Res 6(4): 242-249.

31. Amaechi BT, Higham SM (2002) Quantitative light-induced fluorescence: a potential tool for general dental assessment. J Biomed Opt 7(1): 7-13.

32. Pinelli C, Campos Serra M, de Castro Monteiro Loffredo L (2002) Validity and reproducibility of a laser fluorescence system for detecting the activity of white-spot lesions on free smooth surfaces in vivo. Caries Res 36(1): 19-24.

33. Pretty IA, Hall AF, Smith PW, Edgar WM, Higham SM (2002) The intra- and inter-examiner reliability of quantitative light-induced 
fluorescence (QLF) analyses. Br Dent J 193(2): 105-109.

34. Kuhnisch J, Heinrich-Weltzien R (2004) Quantitative light-induced fluorescence (QLF)--a literature review. Int J Comput Dent 7(4): 325338.

35. Ando M, van Der Veen MH, Schemehorn BR, Stookey GK (2001) Comparative study to quantify demineralized enamel in deciduous and permanent teeth using laser- and light-induced fluorescence techniques. Caries Res 35(6): 464-470.

36. Tranaeus S, Shi XQ, Lindgren LE, Trollsas K, Angmar-Mansson B (2002) In vivo repeatability and reproducibility of the quantitative light-induced fluorescence method. Caries Res 36(1): 3-9.

37.Van der Veen MH, Thomas RZ, Lagerweij MD, Ruben JL, Huysmans, MCDNJM (2002) Non-disclosed and disclosed plaque observed using light induced fluorescence. Caries Res 36: 215.

38. Lee VA, Karthikeyan R, Rawls HR, Amaechi BT (2010) Anti-cariogenic effect of a cetylpyridinium chloride-containing nanoemulsion. J Dent 38(9): 742-749.

39. de Josselin de Jong E, Sundstrom F, Westerling H, Tranaeus S, ten Bosch JJ, et al. (1995) A new method for in vivo quantification of changes in initial enamel caries with laser fluorescence, Caries Res 29(1), 2-7.
40. Turkkahraman H, Sayin MO, Bozkurt FY, Yetkin Z, Kaya S, et al. (2005) Archwire ligation techniques, microbial colonization, and periodontal status in orthodontically treated patients. Angle Orthod 75(2): 231236.

41. Pizzoni L, Ravnholt G, Melsen B (1998) Frictional forces related to self-ligating brackets. Eur J Orthod 20(3): 283-291.

42. Hain M, Dhopatkar A, Rock P (2003) The effect of ligation method on friction in sliding mechanics. Am J Orthod Dentofacial Orthop 123(4): 416-422.

43. Hodgson RJ, Lynch RJ, Watson GK, Labarbe R, Treloar R, et al. (2001) A continuous culture biofilm model of cariogenic responses. J Appl Microbiol 90(3): 440-448.

44. Sissons CH, Cutress TW, Hoffman MP, Wakefield JS (1991) A multistation dental plaque microcosm (artificial mouth) for the study of plaque growth, metabolism, $\mathrm{pH}$ and mineralization. J Dent Res $70(11): 1409-1416$.

45. Dawes C (1993) The ebb and flow of the salivary tide. In: Bowen WH \& Tabak LA (Eds.), Cariology for the nineties. ( $1^{\text {st }}$ edn), University Rochester Press, New York, USA, pp. 133-141. 